\title{
Antioxidant-mediated response of a susceptible papaya cultivar to a compatible strain of Erwinia mallotivora
}

\begin{abstract}
Dieback disease caused by Erwinia mallotivora is the most devastating disease of papaya in Malaysia. Most papaya cultivars here are susceptible to the disease, indicating a compatible relationship between the cultivars and the pathogenic strains of the bacteria. In this study, we evaluated early responses of a susceptible cultivar to a virulent strain of the bacteria by monitoring the disease symptoms, measuring the expression patterns and activities of the oxidative enzymes superoxide dismutase (SOD) and peroxidase (POD), and profiling the leaf proteome. Water-soaked lesions and necrotic spots were evidenced on the inoculated leaves at 6 days post-inoculation (dpi) which were deteriorated rapidly with death at $12 \mathrm{dpi}$. The two oxidative enzymes were responsive to the infection as shown by their induced enzymatic activities and gene expressions in the leaves of the infected plants. The 2D-PAGE profile of the leaf proteome exhibited a significant increase in the intensity of the SOD spot, confirming its involvement in the response. In addition, differential expressions of the protein spots known to be related to stress response, energy production and photosynthesis provided insights into the plant's global response to the infection. Overall, this study suggested that the early response to E. mallotivora inoculation in the susceptible papaya cultivar was likely to be mediated by the antioxidant defense system but the effectiveness of the defense could have been compromised with the down-regulation of important proteins required for normal plant growth and development. This study provides the basis for studying the defect in the defense mechanism that turned this cultivar susceptible to the infection.
\end{abstract}

Keyword: Antioxidant system; Dieback disease; Erwinia mallotivora; Papaya 